Research Paper

\title{
Prognostic Significance of PSMD1 Expression in Patients with Gastric Cancer
}

\author{
Wenjun Xiong ${ }^{1,2^{*}}$, Wei Wang ${ }^{2 *}$, Haipeng Huang ${ }^{2}$, Yuming Jiang ${ }^{1}$, Weihong Guo ${ }^{1}$, Hao Liu ${ }^{1}$, Jiang Y Y ${ }^{1}$, \\ Yanfeng $\mathrm{Hu}^{1}$, Jin $\mathrm{Wan}^{2 \# \bowtie}$, Guoxin $\mathrm{Li}^{1 \# \bowtie}$ \\ 1. Department of General Surgery, Nanfang Hospital, Southern Medical University, Guangdong Provincial Engineering Technology Research Center of \\ Minimally Invasive Surgery, Guangzhou, China \\ 2. Department of Gastrointestinal Surgery, Guangdong Provincial Hospital of Chinese Medicine, the Second Affiliated Hospital of Guangzhou University of \\ Chinese Medicine, Guangzhou, China \\ * Wenjun Xiong and Wei Wang contributed equally to this work and should be both considered as co-first authors. \\ \# Guoxin Li and Jin Wan contributed equally to this work. \\ $\triangle$ Corresponding authors: Guoxin Li, Department of General Surgery, Nanfang Hospital, Southern Medical University, 1838 North Guangzhou Avenue, \\ Guangzhou 510515, China. Jin Wan, Department of Gastrointestinal Surgery, Guangdong Provincial Hospital of Chinese Medicine, Dade Road No.111, 510120, \\ Guangzhou, China. Tel: +86-20-61641681; Fax: +86-20-62787626; E-mail: gzliguoxin@163.com; gdphtcmwanjin@163.com
}

(c) The author(s). This is an open access article distributed under the terms of the Creative Commons Attribution License (https://creativecommons.org/licenses/by/4.0/). See http://ivyspring.com/terms for full terms and conditions.

Received: 2018.11.15; Accepted: 2019.05.21; Published: 2019.07.20

\begin{abstract}
Background: PSMDI has been considered to be involved in many human cancers, but its prognostic significance in gastric cancer (GC) has not been elucidated. The aim of this study was to evaluate the prognostic value of PSMDI expression in tumor tissues of GC patients.

Methods: We retrospectively analyzed the expression of PSMDI in 241 paraffin-embedded GC specimens of the training cohort by immunohistochemistry. The prognostic value of PSMDI expression was assessed using Kaplan-Meier survival curves and multivariate COX regression models. PSMDI expression and other GC-associated risk factors were used to generate two nomograms to evaluate prognosis, and the performance of the two nomograms was assessed with respect to its calibration, discrimination, and clinical usefulness. Further validation was performed using an independent cohort of 170 cases.

Results: High PSMDI expression was significantly associated with decreased disease-free survival (DFS) and overall survival (OS) in GC patients. Furthermore, multivariate Cox proportional hazard analysis demonstrated that PSMDI was an independent prognostic factor for DFS and OS. The two nomograms that were developed by integrating PSMDI expression and the TNM staging system showed better prediction of DFS and OS than TNM staging system alone(C-index for training cohort: 0.708 (95\% Cl:0.670-0.746) and $0.712(0.671-0.752)$, respectively; C-index for validation cohort: 0.704 (0.651-0.757) and $0.711(0.656-0.767)$, respectively). Decision curve analysis demonstrated that the nomograms showed potential for clinical use.

Conclusion: Intratumoral PSMDI expression is a novel independent predictor of DFS and OS in GC patients. In the future, large-scale prospective studies will be necessary to confirm our findings regarding its potential prognostic and therapeutic value for GC patients.
\end{abstract}

Key words: PSMD1, prognostic significance, gastric cancer

\section{Introduction}

Gastric cancer (GC) is the most common cause of cancer deaths worldwide [1]. Despite efforts to improve the survival of patients with GC, satisfactory outcomes have not been achieved. Currently, the prognostic models for $\mathrm{GC}$ are mainly based on the International Union Against Cancer (UICC) Tumor-Node-Metastasis (TNM) staging system. However, the outcomes for patients with a similar 
TNM stage are highly variable because of the inherent heterogeneity [2-5]. Therefore, stratifying patients in the current TNM staging system based on the molecular factors that are involved in gastric carcinogenesis improves the prediction of the clinical outcomes [3].

Proteasomes are multisubunit proteases that mediate the degradation of ubiquitinated proteins [6]. The 19S regulatory particle (19S-RP) of the 26S proteasome directs the ubiquitinated degradation substrates into the proteasome's catalytic $20 \mathrm{~S}$ core particle (20S-CP) of the proteasome. PSMD1 (Rpn2 in yeast) is the largest subunit of 19S-RP. PSMD1is a key structural component of the 19S-RP, which acts as a docking site for other proteasomal subunits. Many tumor suppressor and oncogenic proteinsare regulated by ubiquitin-proteosome mediated protein degradation $[7,8]$. Therefore, it is conceivable that PSMD1 plays an important role in regulating carcinogenesis and cancer progression. Recent studies show that PSMD1 is upregulated in anaplastic thyroid carcinoma (ATC) and breast cancer tissues and shows potential as a novel therapeutic target [9, 10]. Okumura et al. showed that PSMD1as a potential critical gene that may regulate cell proliferation and cell-cycle progression by mediating p53 protein degradation in breast cancer cells [10]. However, the role and prognostic potential of PSMD1 in GC are not yet known.

In this study, through quantitative polymerase chain reaction (qPCR) and immunohistochemistry assays, we found that GC tissues showed high PSMD1 protein expression. We further demonstrated that closely associations existed between PSMD1 levels and clinicopathological factors. Therefore, we postulate that PSMD1 is a potential target for diagnosis and clinical treatment of GC. We also developed two predictive nomograms to assess the risk score for overall survival (OS) and disease-free survival (DFS) of GC patients by integrating parameters such as PSMD1 expression, tumor depth, lymph node metastasis and distant metastasis.

\section{Materials and Methods}

\section{Patients and tissue specimens}

Thirty-six fresh frozen GC and corresponding nontumoral gastric mucosa tissue samples were taken from patients with GC within 30 min after resection, and then snap-frozen in liquid nitrogen and stored at $-80{ }^{\circ} \mathrm{C}$ until use (Table S1). The thirty-six patients were availability of hematoxylin and eosin slides with invasive tumor components, availability of complete clinicopathologic characteristics, no preoperative anticancer treatment, and more than 15 examined lymph nodes. Furthermore, we enrolled two independent panels of formalin-fixed paraffin-embedded (FFPE) specimens derived from 411 GC patients for this study. This included a training cohort of 241 patients with incident, primary, biopsy-confirmed GC, diagnosed from June 2006 to April 2008 at Nanfang Hospital of Southern Medical University (Guangzhou, China). Inclusion criteria were availability of hematoxylin and eosin slides with invasive tumor components, availability of follow-up data and clinicopathologic characteristics, no history of cancer treatment, more than 15 examined lymph nodes, and appropriate patient informed consent. We excluded patients if formalin-fixed paraffin-embedded (FFPE) tumor and normal samples from the initial diagnosis were unavailable or if they had received previous treatment with any anticancer therapy. Two independent pathologists reassessed all these samples. The internal validation cohort included an additional 170 patients, with the same criteria as above, diagnosed from May 2008 to May 2009 at Nanfang Hospital of Southern Medical University. TNM staging was reclassified according to the AJCC staging manual (seventh edition). Two independent pathologists reassessed all these samples. This study was approved by the research ethics committee at Nanfang Hospital of Southern Medical University and the need to obtain informed consent was waived because of the retrospective nature of the study.

\section{RNA extraction and quantitative PCR}

Total RNA was extracted with TRIzol reagent (TaKaRa, Dalian China) and cDNA was synthesized from $5 \mathrm{ng}$ of total RNA using the mRNA reverse transcription kit (TaKaRa, Dalian China). We performed real-time PCR to quantitate mRNA expression as described in the protocol supplied with SYBR Premix Ex Taq (Takara) using a LightCycler 480 v.1.5 system (Roche). PSMD1 gene expression were normalized to GAPDH gene expression (internal control) using the 2- $\Delta \Delta \mathrm{Ct}$ method $[11,12]$.

\section{Immunohistochemistry (IHC)}

FFPE samples were subjected to IHC as previously described [13-15]. The samples were cut into $4 \mu \mathrm{m}$-thick sections, de-waxed in xylene, and rehydrated in decreasing concentrations of ethanol. Prior to staining, sections were blocked with endogenous peroxidase (prepared in $1 \%$ $\mathrm{H}_{2} \mathrm{O}_{2}$ /methanol solution) for $10 \mathrm{~min}$ and then microwaved for $30 \mathrm{~min}$ in $10 \mathrm{mM}$ citrate buffer, pH6.0. Then, the sections were blocked using $10 \%$ normal rabbit serum for $30 \mathrm{~min}$. The slides were incubated overnight withanti-PSMD1 antibody (1:200 
dilution; ab140677; Abcam, Cambridge, MA) at $4^{\circ} \mathrm{C}$, followed by incubation with an amplification system with a labeledpolymer/HRP (EnVision ${ }^{\mathrm{TM}}$, DakoCytomation, Denmark) for $30 \mathrm{~min}$. The sections were developed with $0.05 \%$ 3, 3'-diaminobenzidine tetrahydrochloride (DAB) and counterstained with modified Harris hematoxylin.

\section{Evaluation of IHC staining}

Two experienced pathologists who were blinded to the clinical parameters and outcomes for each patient independently reviewed the IHC stained sections. Each section was scored by randomly selecting and examining 10 fields in the tumor region with a high-power microscope. Their results were in complete agreement in approximately $90 \%$ of the cases. A third pathologist was consulted when different opinions arose between the two primary pathologists. If the third pathologist agreed with one of them, then that value was selected. If the conclusion by the third pathologist was completely different, then the three of them would work collaboratively to find a common answer. PSMD1 expression in each specimen was evaluated by determining a semi-quantitative $\mathrm{H}$ score, which was calculated by multiplying the result of a 4 -stepscale $(0$ = negative, $0.5=$ weak staining, $1=$ moderate staining, 1.5 = strong staining) and the fraction of positively stained cells that ranged from 0 to $100 \%[16,17]$. The $\mathrm{H}$-scores for PSMD1 staining were dichotomized at the median to classify GC patients into high and low PSMD1 expression groups.

\section{Construction of the Nomograms}

In the training cohort, survival curves for different variables were generated using the Kaplan-Meier estimates and were compared using the log-rank test. Variables that achieved statistical significance with a $P<0.05$ were subjected to multivariable analyses using the Cox regression model. Statistical analysis to identify independent prognostic factors was performed by using the SPSS 19.0 software for Windows (SPSS, Chicago, IL). Based on the results of the multivariable analysis, two nomograms were established using the survival and rms package of the $\mathrm{R} \quad 3.4 .0$ software (http://www.r-project.org). Backward step-wise selection was determined by the likelihood ratio test using Akaike's information criterion as the stopping rule [18].

\section{Validation and Calibration of the Nomograms}

The performance of the two nomograms for predicting survival outcomes was tested in the validation cohort by calculating the concordance index (C-index) [19]. The value of the C-index ranged from 0.5 to 1.0 , with 0.5 indicating a random chance and 1.0 indicating a perfect clear-cut ability to correctly determine the survival outcome with the model. The nomograms were calibrated for 1-, 3-, and 5 -year DFS and OS by comparing predicted survival with observed survival after bias correction.

\section{Clinical Use}

Decision curve analysis was performed to determine the clinical usefulness of the nomograms by quantifying the net benefits at different threshold probabilities [20,21].

\section{Risk Group Stratification Based on the Nomogram}

The composite scoring of the nomograms was divided into three risk groups using X-tile [22], which accurately discriminated patients with good, intermediate, and poor prognosis.

\section{Statistical analysis}

We analyzed the statistical data for two groups by using the $t$ test for continuous variables and $\chi^{2}$ test for categorical variables. The DFS and OS were defined as the number of months from the date of surgery to the date of regional recurrence or distant metastasis (for DFS) and death or final clinical follow-up (for OS). The Kaplan-Meier method and the log-rank test were used to estimate DFS and OS. Multivariate Cox proportional hazards regression analysis was performed for all variables that were significant in the univariate analysis. The statistical tests were performed using the $\mathrm{R}$ (version 3.4.0)and SPSS (version 19.0) software packages. All statistical tests were two-sided, and $P<0.05$ was regarded as statistically significant.

\section{Results}

\section{Overexpression of PSMDI in human GC}

To elucidate the role of PSMD1 in the initiation and progression of GC, we first analyzed its expression by real-time PCR in 36 GC biopsies and matched adjacent nontumoral tissues at the mRNA level. mRNA level analysis of PSMD1 expression in matched nontumoral and tumor tissues showed that PSMD1 was upregulated in the majority of GC tissues compared to their nontumoral counterparts (25/36; 69.4\%) (Fig. S1), and PSMD1 protein levels were also significantly higher in tumor tissues (Fig. S2). Furthermore, compared with the nontumoral PSMD1 density, intratumoral PSMD1 expression was also higher $(P<0.0001$; Fig. 1A-B), as shown by IHC findings. Table 1 lists the detailed clinicopathological characteristics of the GC patients in the training and validation cohorts. The expression of PSMD1 was 
much higher in advanced stage GC [stages I-II $(\mathrm{n}=80)$ vs. stages III-IV ( $\mathrm{n}=161), P=0.0006]$. Furthermore, the percentage of patients with high intratumoral

A
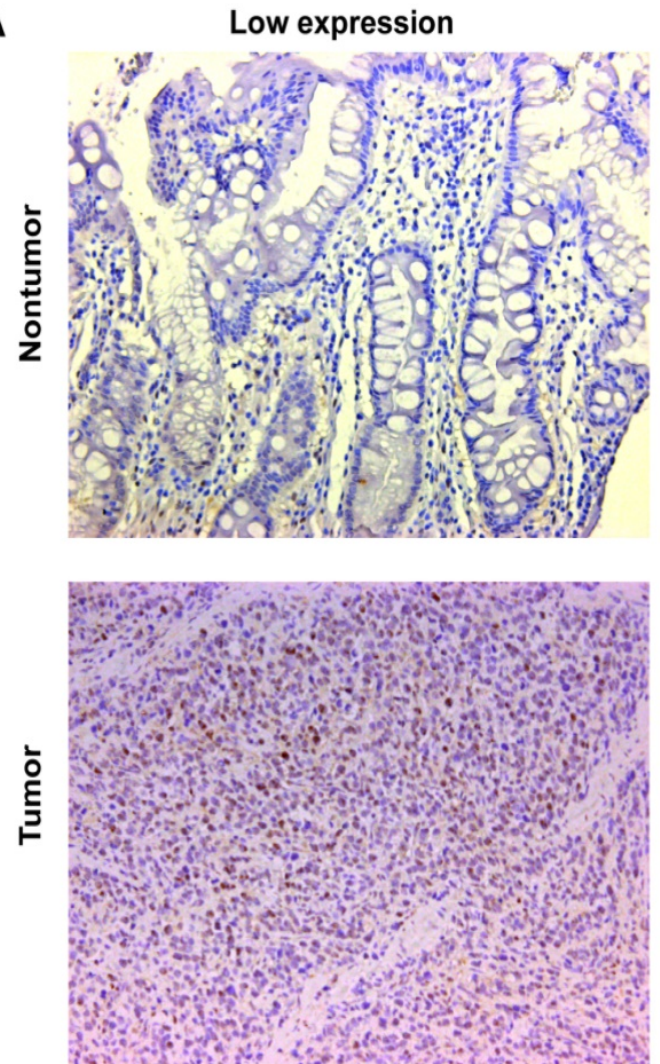

B

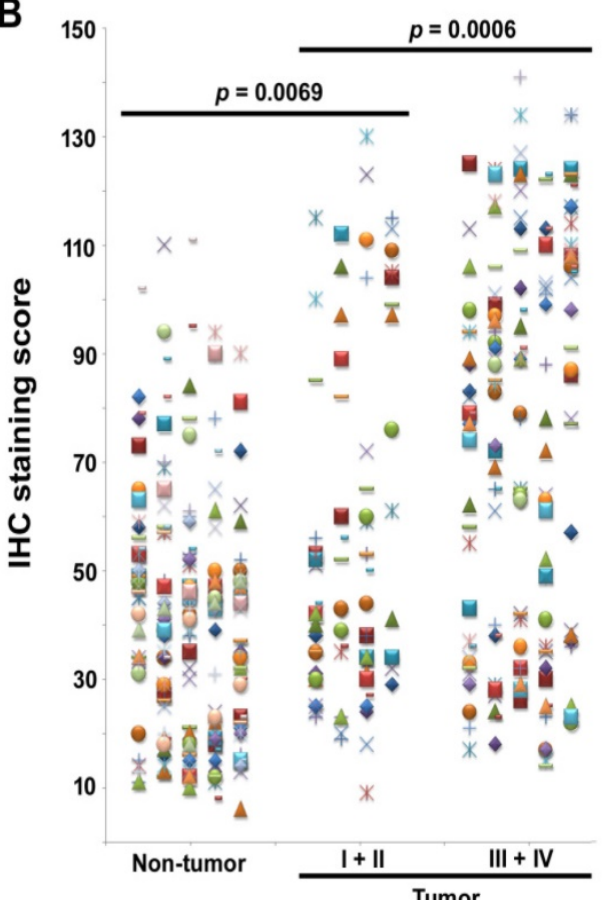

PSMD1 expression increased moderately from TNM stages I to IV, suggesting its association with disease progression (Fig. 1C, Table 1).
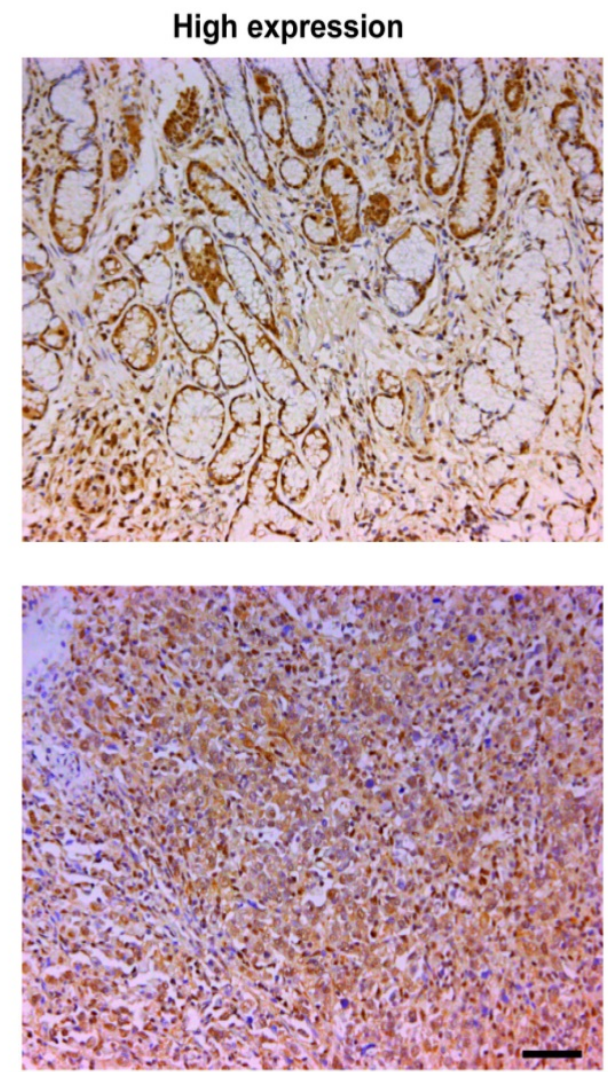

C

PSMD1 high expression PSMD1 low expression

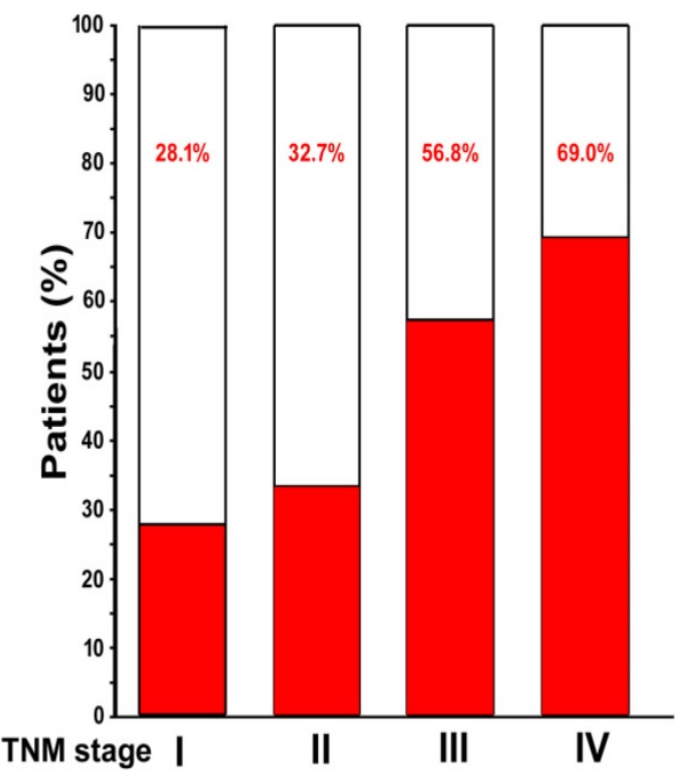

Figure 1. PSMDI expression in GC tissues. (A) Representative IHC images of PSMDI expression in nontumor and tumor tissues of GC patients. Left panel: low expression; right panel: high expression. (B) Scatter plots for IHC staining score in unpaired nontumor $(n=241)$ and tumor $(n=241)$ tissues from the training cohort. $P$ value was determined using the nonparametric Mann-Whitney test. c Increased percentage of GC patients belonging to TNM stages I-IV show higher intratumoral PSMDI expression suggesting its association with disease progression (data from the training and validation cohorts). Scale bar, $100 \mu \mathrm{m}$. 
Table 1. Clinical characteristics of patients according to PSMDI in the training and validation cohorts.

\begin{tabular}{|c|c|c|c|c|c|c|c|c|}
\hline \multirow[t]{2}{*}{ Variables } & \multicolumn{4}{|c|}{ Training cohort $(n=241)$} & \multicolumn{4}{|c|}{ Validation cohort $(n=170)$} \\
\hline & $\mathrm{N}$ & low PSMD1(\%) & high PSMD1(\%) & $\mathrm{p}$ value & $\mathrm{N}$ & low PSMD1(\%) & high PSMD1(\%) & $\mathrm{p}$ value \\
\hline Gender & & & & 0.141 & & & & 0.219 \\
\hline Female & 68 & $29(42.6 \%)$ & $39(57.4 \%)$ & & 80 & $44(55.0 \%)$ & $36(45.0 \%)$ & \\
\hline Male & 173 & $92(53.2 \%)$ & $81(46.8 \%)$ & & 90 & $41(45.6 \%)$ & $49(54.4 \%)$ & \\
\hline Age(years) & & & & 0.941 & & & & 0.442 \\
\hline$<60$ & 138 & $69(50.0 \%)$ & $69(50.0 \%)$ & & 91 & $48(52.7 \%)$ & $43(47.3 \%)$ & \\
\hline$\geqq 60$ & 103 & $52(50.5 \%)$ & $51(49.5 \%)$ & & 79 & $37(46.8 \%)$ & $42(53.2 \%)$ & \\
\hline Tumor location & & & & 0.125 & & & & 0.003 \\
\hline Higher & 49 & $32(65.3 \%)$ & $17(34.7 \%)$ & & 34 & $13(38.3 \%)$ & $21(61.8 \%)$ & \\
\hline Moderate & 39 & $19(48.7 \%)$ & $20(51.3 \%)$ & & 32 & $12(37.5 \%)$ & $20(62.5 \%)$ & \\
\hline Lower & 119 & $54(45.4 \%)$ & $65(54.6 \%)$ & & 84 & $54(64.3 \%)$ & $30(35.7 \%)$ & \\
\hline Whole & 34 & $16(47.1 \%)$ & $18(52.9 \%)$ & & 20 & $6(30.0 \%)$ & $14(70.0 \%)$ & \\
\hline Size & & & & 0.108 & & & & 0.039 \\
\hline$<4$ & 123 & $68(55.3 \%)$ & $55(44.7 \%)$ & & 63 & $38(60.3 \%)$ & $25(39.7 \%)$ & \\
\hline$\geqq 4$ & 118 & $53(44.9 \%)$ & $65(55.1 \%)$ & & 107 & $47(43.9 \%)$ & $60(56.1 \%)$ & \\
\hline Differentiation status & & & & 0.254 & & & & 0.02 \\
\hline Well & 44 & $27(61.4 \%)$ & $17(38.6 \%)$ & & 20 & $5(25.0 \%)$ & $15(75.0 \%)$ & \\
\hline Moderate & 80 & $39(48.8 \%)$ & $41(51.3 \%)$ & & 55 & $25(45.5 \%)$ & $30(54.5 \%)$ & \\
\hline Poor and undifferentiated & 117 & $55(47.0 \%)$ & $62(53.0 \%)$ & & 95 & $55(57.9 \%)$ & $40(42.1 \%)$ & \\
\hline CEA & & & & 0.015 & & & & 0.002 \\
\hline Elevated & 73 & $28(38.4 \%)$ & $45(61.6 \%)$ & & 46 & $14(30.4 \%)$ & $32(69.6 \%)$ & \\
\hline Normal & 168 & $93(55.4 \%)$ & $75(44.6 \%)$ & & 124 & $71(57.3 \%)$ & $53(42.7 \%)$ & \\
\hline CA199 & & & & 0.046 & & & & 0.007 \\
\hline Elevated & 74 & $30(40.5 \%)$ & $44(59.5 \%)$ & & 41 & $13(31.7 \%)$ & $28(68.3 \%)$ & \\
\hline Normal & 167 & $91(54.5 \%)$ & $76(45.5 \%)$ & & 129 & $72(55.8 \%)$ & $57(44.2 \%)$ & \\
\hline Depth of invasion & & & & 0.007 & & & & 0.038 \\
\hline $\mathrm{T} 1$ & 14 & $11(78.6 \%)$ & $3(21.4 \%)$ & & 20 & $15(75.0 \%)$ & $5(25.0 \%)$ & \\
\hline $\mathrm{T} 2$ & 36 & $25(69.4 \%)$ & $11(30.6 \%)$ & & 16 & $9(56.3 \%)$ & $7(43.7 \%)$ & \\
\hline T3 & 20 & $11(55.0 \%)$ & $9(45.0 \%)$ & & 6 & $5(83.3 \%)$ & $1(16.7 \%)$ & \\
\hline $\mathrm{T} 4 \mathrm{a}$ & 131 & $59(45.0 \%)$ & $72(55.0 \%)$ & & 91 & $41(45.1 \%)$ & $50(54.9 \%)$ & \\
\hline $\mathrm{T} 4 \mathrm{~b}$ & 40 & $15(37.5 \%)$ & $25(62.5 \%)$ & & 37 & $15(40.5 \%)$ & $22(59.5 \%)$ & \\
\hline Lymph node metastasis & & & & 0.003 & & & & 0.497 \\
\hline No & 53 & $38(71.7 \%)$ & $15(28.3 \%)$ & & 41 & $23(56.1 \%)$ & $18(43.9 \%)$ & \\
\hline N1 & 60 & $30(50.0 \%)$ & $30(50.0 \%)$ & & 45 & $19(42.2 \%)$ & $26(57.8 \%)$ & \\
\hline N2 & 69 & $25(36.2 \%)$ & $44(63.8 \%)$ & & 43 & $20(46.5 \%)$ & $23(53.5 \%)$ & \\
\hline $\mathrm{N} 3 \mathrm{a}$ & 39 & $20(51.3 \%)$ & $19(48.7 \%)$ & & 24 & $12(50.0 \%)$ & $12(50.0 \%)$ & \\
\hline $\mathrm{N} 3 \mathrm{~b}$ & 20 & $8(40.0 \%)$ & $12(60.0 \%)$ & & 17 & $11(64.7 \%)$ & $6(35.3 \%)$ & \\
\hline Metastasis & & & & 0.036 & & & & 0.115 \\
\hline M0 & 215 & $113(52.6 \%)$ & $102(47.4 \%)$ & & 154 & $80(51.9 \%)$ & $74(48.1 \%)$ & \\
\hline M1 & 26 & $8(30.8 \%)$ & $18(69.2 \%)$ & & 16 & $5(31.3 \%)$ & $11(68.8 \%)$ & \\
\hline TNM stage & & & & $<0.0001$ & & & & $<0.0001$ \\
\hline I & 19 & $14(73.7 \%)$ & $5(26.3 \%)$ & & 32 & $23(71.9 \%)$ & $9(28.1 \%)$ & \\
\hline II & 59 & $42(71.2 \%)$ & $17(28.8 \%)$ & & 101 & $68(67.3 \%)$ & $33(32.7 \%)$ & \\
\hline III & 137 & $57(41.6 \%)$ & $80(58.4 \%)$ & & 236 & $102(43.2 \%)$ & $134(56.8 \%)$ & \\
\hline IV & 26 & $8(30.8 \%)$ & $18(69.2 \%)$ & & 42 & $13(31.0 \%)$ & $29(69.0 \%)$ & \\
\hline
\end{tabular}

\section{High expression of PSMDI is associated with poor clinical outcomes}

To determine the prognostic value of PSMD1 expression in GC patients, Kaplan-Meier survival analysis was performed to analyze DFS and OS based on PSMD1 expression. In the training cohort, PSMD1 expression in non-tumor tissue was not associated with DFS and OS (Fig. 2A). However, patients with high intratumoral PSMD1 expression were associated with lower 5-year DFS and OS than patients with low intratumoral PSMD1 expression (5-year DFS: 17.5\% vs. $42.1 \%$; hazard ratio (HR) $=1.977$ (1.428-2.739); 5-year OS: $24.2 \%$ vs. $48.9 \%$; HR=1.992 (1.417-2.801); all $\mathrm{P}<0.001$; Fig. 2B). We obtained similar results in the validation cohort (Fig. 2C), which confirmed the prognostic significance of PSMD1 expression in different populations of GC patients. Multivariate analysis showed that PSMD1 was an independent prognostic factor for predicting DFS and OS in GC patients (Table 2 and S2-3). When stratified by clinicopathological risk factors, PSMD1 remained a clinically relevant prognostic marker (Fig. S3-4).

\section{Development and validation of nomograms for predicting prognosis of GC patients}

According to all significant independent risk factors for survival of GC patients, we established two nomograms using the multivariate Cox regression model to predict DFS and OS of GC patients (Fig. 3). The nomograms can be interpreted by adding the points assigned to each significant variable that are indicated at the top of the scale. The total points can then be used to predict 1-, 3- and 5-year DFS and OS for a patient in the lowest scale [23]. In the training cohort, the C-indexes for DFS and OS prediction were 0.708 (95\% CI: $0.670-0.746)$ and $0.712 \quad(95 \%$ CI: $0.671-0.752)$, respectively. Recalibration was not 
required because the calibration curves for the two nomograms (Fig. 4A-B) did not deviate from the reference line. In the validation cohort, the C-indexes for DFS and OS prediction were 0.704 (0.651-0.757) and 0.711 (0.656-0.767), respectively. The calibration curves yielded good agreement between the predicted and observed outcomes for both DFS and OS (Fig. 4C-D). Furthermore, the performances of the nomograms for DFS and OS in both the training and validation cohorts were superior relative to the TNM staging system alone (Fig. S5). The C-indexes of TNM stage for DFS and OS prediction were 0.681 $(0.648-0.715)$ and $0.678(0.640-0.716)$ in the training cohort, and 0.660 (0.610-0.709) and 0.661 (0.610-0.712) in the validation cohort, respectively. Using the X-tile, the composite scoring was divided into three risk groups that accurately discriminated between patients with good, intermediate, and poor prognosis (Fig. 5 and Fig. S6).

\section{A PSMD1 in Nontumor (Training Cohort)}
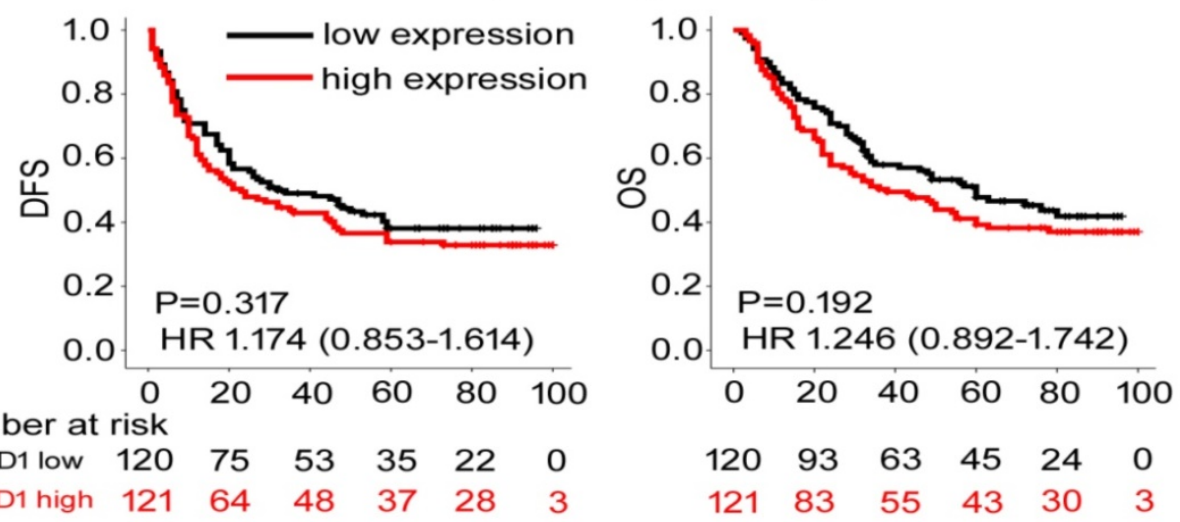

\section{B PSMD1 in Tumor (Training Cohort)}
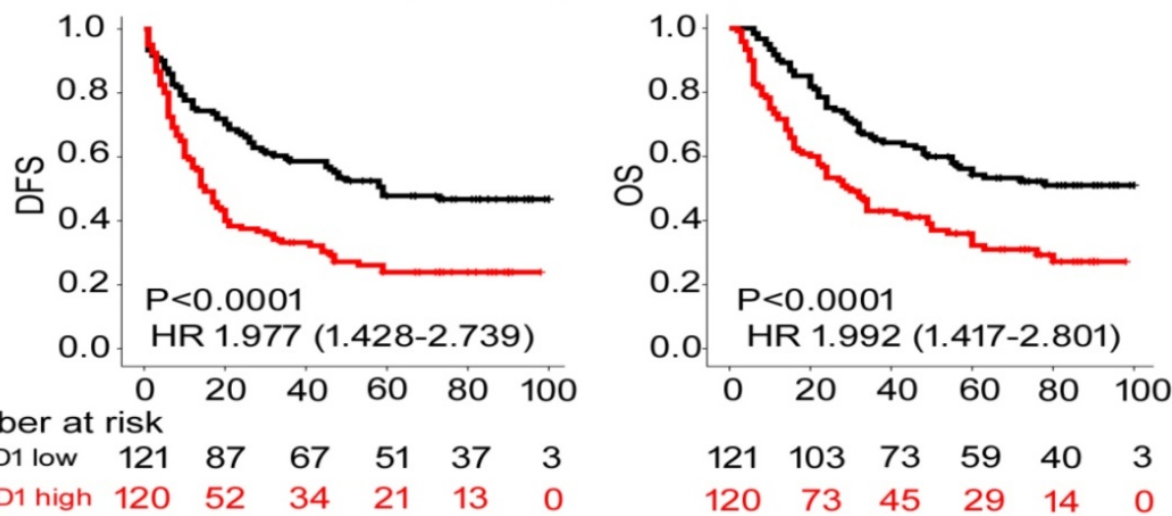

C PSMD1 in Tumor (Validation Cohort)
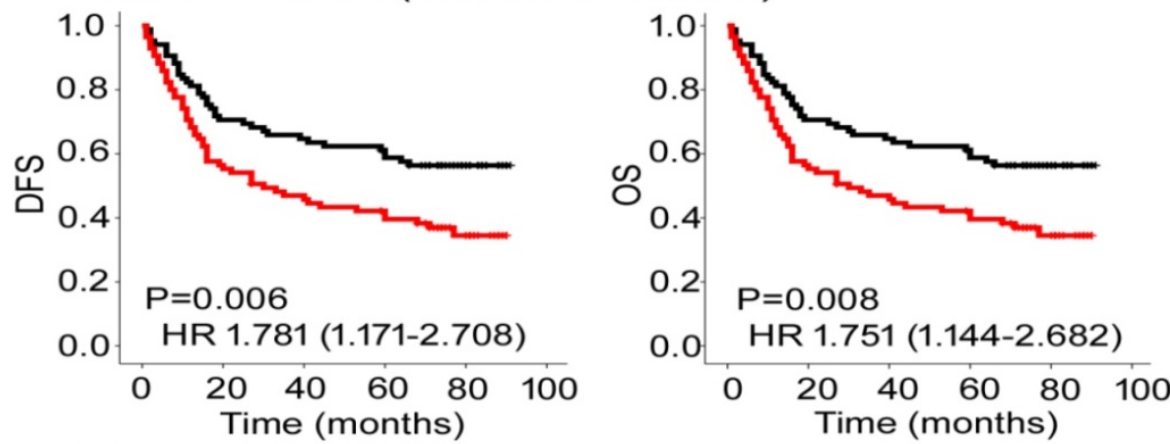

Number at risk

$\begin{array}{llllllllllllll}\text { PSMD1 low } & 85 & 60 & 55 & 51 & 21 & 0 & 85 & 69 & 57 & 53 & 21 & 0 \\ \text { PSMD1 high } & 85 & 48 & 39 & 33 & 14 & 0 & & 85 & 54 & 43 & 35 & 16 & 0\end{array}$

Figure 2. Kaplan-Meier survival analyses of disease-free survival (DFS) and overall survival (OS) in the training and validation cohorts of GC patients classified according to PSMD1 expression in (A) nontumor and tumor $(\mathbf{B}, \mathbf{C})$ tissues. 
A

Points

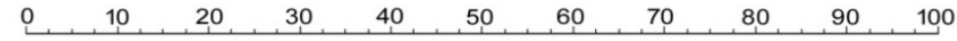

PSMD1

Depth of invasion

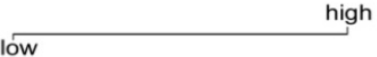

Lymph node metastasis

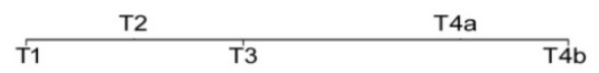

Metastasis

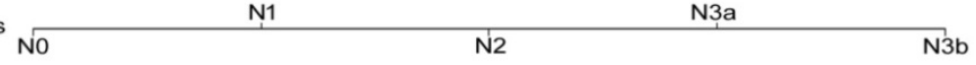

Metastasis

MO

M1

Total points

\begin{tabular}{lllllll}
\hline 50 & 100 & 150 & 200 & 250 & 300 & 350
\end{tabular}

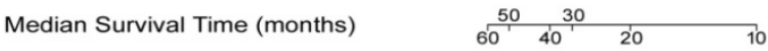

$\begin{array}{lllllllll}\text { Probability of 1-year DFS } & 0.9 & 0.8 & 0.7 & 0.6 & 0.5 & 0.3 & 0.2 & 0.1\end{array}$

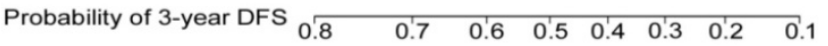

Probability of 5-year DFS

$\begin{array}{lllllll}0.7 & 0.6 & 0.5 & 0.4 & 0.3 & 0.2 & 0.1\end{array}$

B

Points

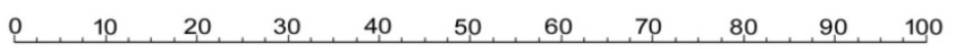

PSMD1

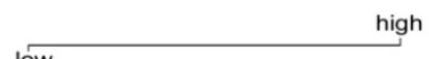

Depth of invasion

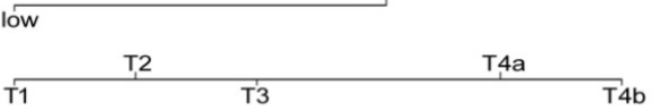

Lymph node metastasi

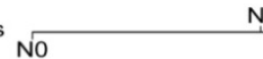
N3a

Metastasis

Mo

M1

Total points

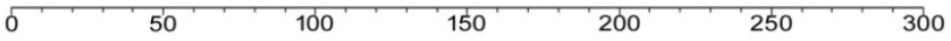

Median Survival Time (months)

Probability of 1-year OS

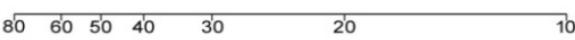

Probability of 3-year OS

$\begin{array}{lllllll}0.9 & 0.8 & 0.7 & 0.6 & 0.5 & 0.4 & 0.3\end{array}$

Probability of 5-year OS

$\begin{array}{llllllll}0.8 & 0.7 & 0.6 & 0.5 & 0.4 & 0.3 & 0.2 & 0.1 \\ 0.7 & 0.6 & 0.5 & 0.4 & 0.3 & 0.2 & 0.1 & \end{array}$

Figure 3. Nomogram for predicting DFS and OS. The grade of the patient is indicated on the grade axis and a straight line is drawn upwards to the point's axis to determine the number of points received by the patient towards survival for his/her grade. This process is repeated for the other axes to determine the sum of the points received for each predictor. A straight line is drawn down to the survival-probability axis to determine the probability of the patient surviving gastric cancer.

Table 2. Multivariable Cox regression analysis of disease-free survival and overall survival.

\begin{tabular}{lllll}
\hline \multirow{2}{*}{ Variables } & \multicolumn{2}{l}{ Disease-free survival } & Overall survival & \\
\cline { 2 - 5 } & HR $(95 \% \mathrm{CI})$ & $\mathrm{p}$ value & HR $(95 \% \mathrm{CI})$ & $\mathrm{p}$ value \\
\hline PSMD1 (high vs. low) & $\mathbf{1 . 6 0 3} \mathbf{( 1 . 1 3 1 - 2 . 2 7 1 )}$ & $\mathbf{0 . 0 0 8}$ & $\mathbf{1 . 5 8 4}(\mathbf{1 . 0 9 6 - 2 . 2 8 9})$ & $\mathbf{0 . 0 1 4}$ \\
Depth of invasion & & $<0.001$ & & 0.003 \\
T1 & Reference & & Reference & \\
T2 & $2.172(0.815-5.785)$ & 0.121 & $5.172(1.203-22.23)$ & 0.027 \\
T3 & $0.907(0.274-3.006)$ & 0.874 & $2.187(0.437-10.94)$ & 0.341 \\
T4a & $1.811(0.719-4.559)$ & 0.208 & $4.255(1.029-17.59)$ & 0.046 \\
T4b & $4.166(1.573-11.034)$ & 0.004 & $7.048(1.645-30.19)$ & 0.009 \\
Lymph node metastasis & & 0.002 & & 0.036 \\
N0 & Reference & & Reference & \\
N1 & $1.559(0.895-2.716)$ & 0.117 & $1.558(0.865-2.805)$ & 0.140 \\
N2 & $1.933(1.130-3.306)$ & 0.016 & $1.743(0.982-3.094)$ & 0.058 \\
N3a & $2.622(1.470-4.678)$ & 0.001 & $2.293(1.240-4.241)$ & 0.008 \\
N3b & $3.282(1.698-6.343)$ & 0.0004 & $2.796(1.373-5.694)$ & 0.005 \\
Metastasis (M1 vs. M0) & $2.537(1.562-4.121)$ & 0.0002 & $2.028(1.198-3.434)$ & 0.008 \\
\hline
\end{tabular}

\section{Clinical Use}

The decision curve analysis for the two nomograms is shown in Fig. 6. The decision curve showed that if the threshold probability of a patient or doctor was $>10 \%$, predicting the 1-, 3-, 5-year DFS and OS was more accurate using the two nomograms than either the treat-all-patients or treat-none schemes.

\section{Discussion}

To our knowledge, this is the first study to analyze the potential prognostic value of PSMD1 in GC. We observed variable expression of PSMD1 in GC tissues, and positive correlation of PSMD1 
expression with TNM stage in tumor tissues. Moreover, high expression of PSMD1 indicated a more aggressive tumor phenotype and was associated with poor DFS and OS.

The 26S proteasome is a multicatalytic proteinase complex that is composed two complexes (20S core and 19S regulator). The PSMD1 gene encodes the largest non-ATPase subunit of the $19 \mathrm{~S}$ regulator lid, which is responsible for substrate recognition and binding. Ryu et al. showed that PSMD1 SUMOylation controls proteasome composition and function, thereby providing a mechanism for regulation of ubiquitin-mediated protein degradation through the SUMO pathway [24]. Jonker et al. revealed that PSMD1was significantly upregulated in ATC and showed potential as a therapeutic target [13]. Deng et al. showed high PSMD1 expression (>3-fold) in breast cancer tissues
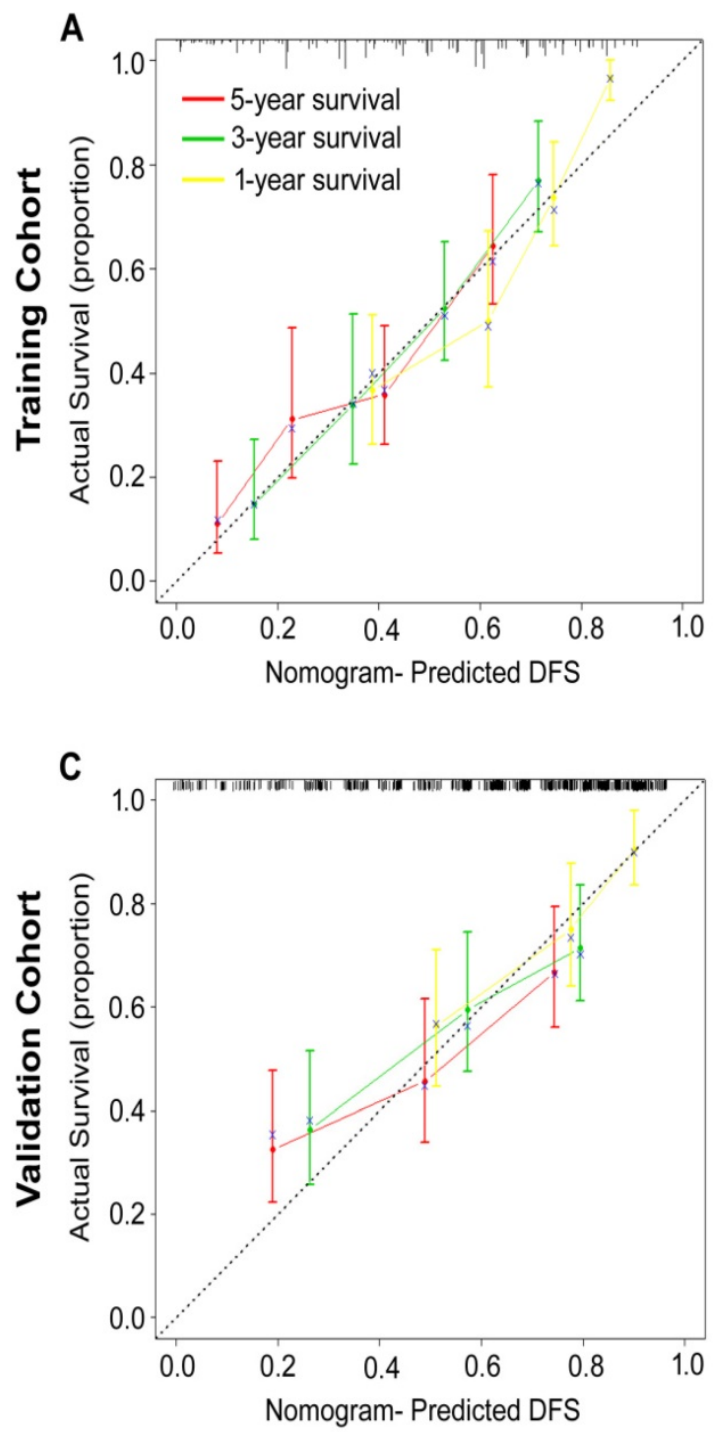

compared to adjacent normal tissues [25]. Wang et al. used cDNA microarray analysis to compare gene expression profiles of acute promyelocytic leukemia cell line NB4 before and after (12 h) regular treatment and demonstrated high PSMD1 expression in the untreated cell line; this suggested a critical role for PSMD1 in the apoptosis and partial differentiation of NB4 cells [26]. In addition, a group of genes encoding proteasomal subunits (PSMA1, PMSE1, PSMD1 and PMSD8) that showed hypomethylated promoters were upregulated in pre-eclampsia [27]. Okumura et al. showed that PSMD1 was associated with poor prognosis of breast cancer patients based on the analyses of a clinical dataset (http://kmplot.com). Lagadecet al. showed that weak PSMD1 expression in the head and neck cancer cells predicts unfavorable outcomes post-radiotherapy [28]. In the present study, we initially showed higher PSMD1expression in GC
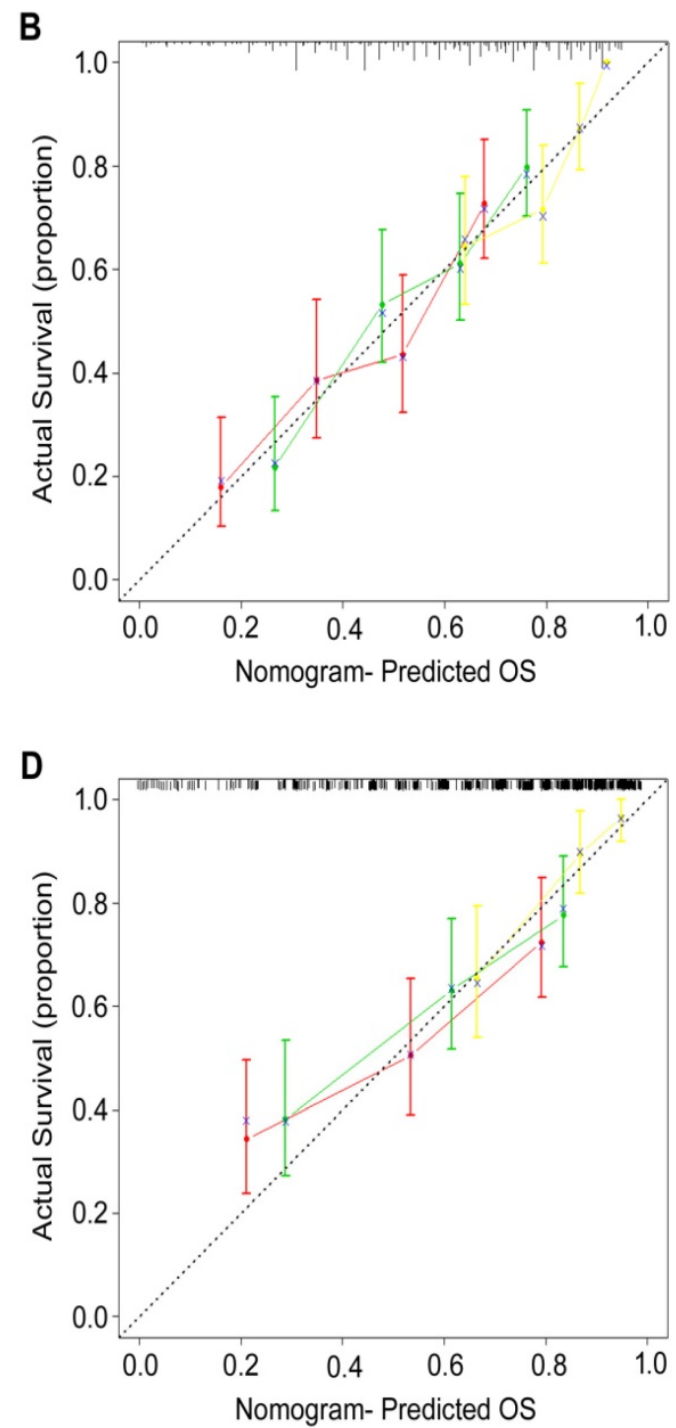

Figure 4. Calibration curves for the nomogram-predicted probability of 1-, 3- and 5-year (A, C) DFS and (B, D) OS of GC patients in the training and validation cohorts. The nomogram-predicted OS and DFS values are plotted on the x-axis, and the actual OS and DFS values are plotted on the $y$-axis. The dotted line represents an ideal nomogram, and the solid blue line represents the current nomogram. The vertical bars represent $95 \% \mathrm{Cls}$, and the $\times$ 's represent bootstrap-corrected estimates. 
tissues than in the surrounding nontumor mucosal tissues by performing qRT-PCR fresh-frozen GC tissues and IHC in FFPE GC specimens. Moreover, high PSMD1 expression was associated with poor prognosis of GC patients.

The proteasome inhibitors, carfilzomib and bortezomib, inhibit ATC tumor growth, both in vitro and in vivo [7, 8]. Bortezomib also induced redifferentiation of ATC cells and increased their iodine uptake. The activity of the ubiquitin-proteasome system is obviously enhanced in many types of human cancer, and its selective inhibition is a potential target for treating human cancers [8, 25]. The knockdown of proteasome $26 \mathrm{~S}$ subunit PSMD1 markedly reduced the proliferation of4-hydroxytamoxifen (OHT) resistant MCF-7 (OHTR) breast cancer cells [10]. Notably, the knockdown of PSMD1 resulted in accumulation of the p53 protein and subsequent cell cycle arrest. Furthermore, in accordance with p53accumulation,
PSMD1 silencing also upregulated p21 and SFN, the target genes of p53. Therefore, PSMD1 may play a role in the development of tamoxifen resistance in breast cancer cells. Our finding provides a new insight for the mechanism underlying the therapeutic resistance of gastric cancer and identifies PSMD1 as a potential prognostic and therapeutic target.

Although several prognostic biomarkers have recently been identified for gastric cancer recently [14, 17], TNM staging remains the most commonly used system to predict survival for GC patients. However, GC patients within the same TNM stage demonstrate different cellular, genetic, and clinicopathological characteristics. Moreover, their survival is highly variable, and TNM staging may not be sufficient to accurately predict GC patients survival [2]. Nomograms have been developed to evaluate a large number of significant clinicopathological factors to improve prognostic prediction of individual patients and to provide a more individualized staging system.

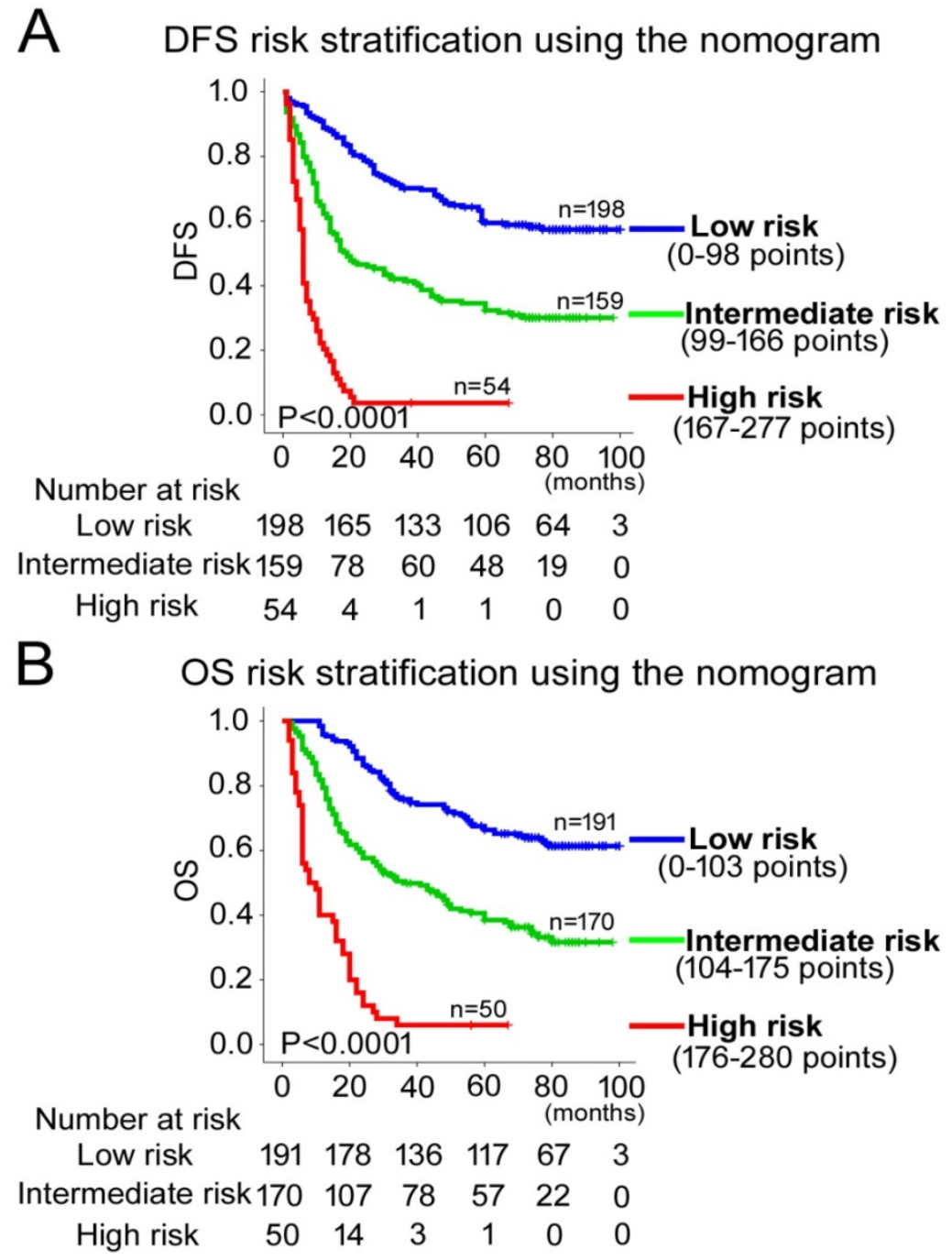

Figure 5. Kaplan-Meier survival analyses of OS and DFS for the three risk groups. The entire patient population was divided into 3 subgroups based on the total number of points obtained in the (A) DFS and (B) OS nomograms. 
Improved prediction of individual outcomes would be useful for counseling patients, personalized treatment, and scheduling patient follow-ups [29]. In our study, PSMD1 expression demonstrates sufficient discriminatory power in most subgroups of different clinicopathological types based on Kaplan-Meier survival curves and univariate COX stratification analysis (Fig. S1-2). Furthermore, multivariate COX regression analysis confirmed the independent prognostic value of PSMD1 expression, which could be integrated with the TNM staging system in the nomogram (Table 2). Nomograms were validated using calibration plots and the C-index. The nomograms performed well with good discrimination and calibration, and the C-index for DFS and OS was satisfactory. AUC and C-index values indicated that the nomogram performed better than the TNM stage
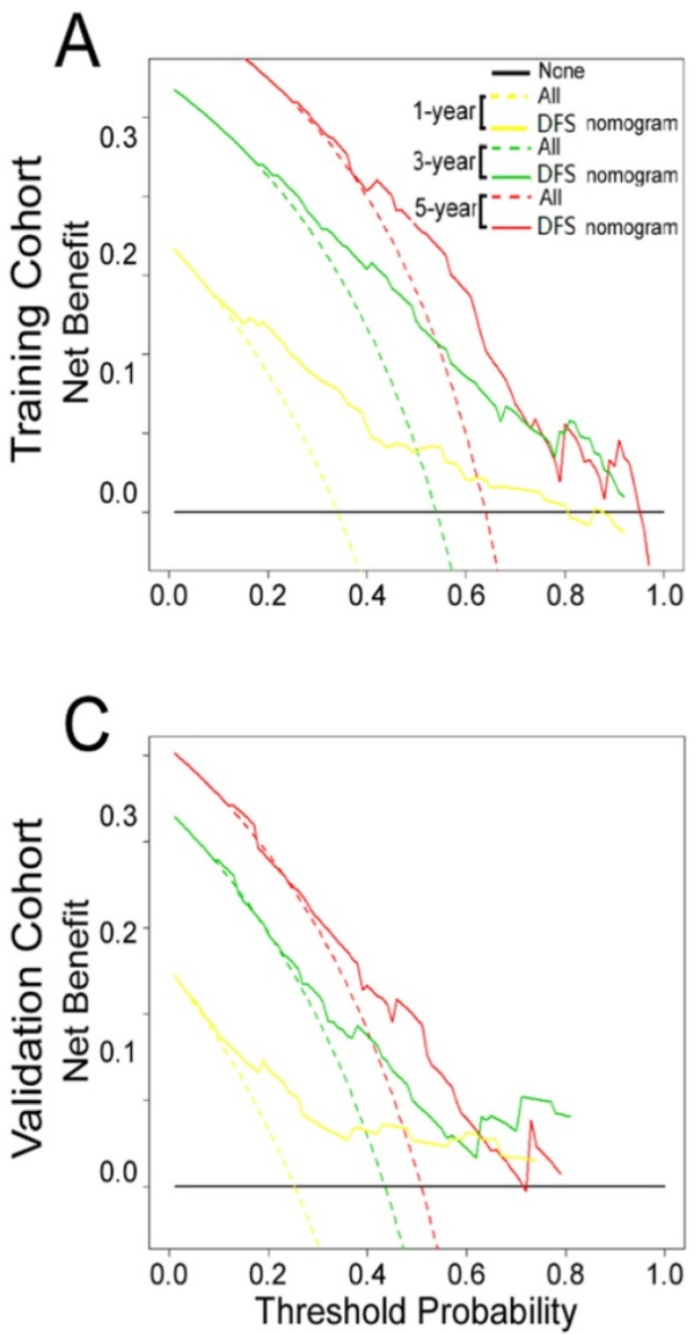

alone (Fig. S3). However, to overcome the limitations of our study as a result of its retrospective design and the relatively small size of the patient population, we propose that a large-scale, multicenter, prospective study is needed to validate our results.

In conclusion, our study demonstrates PSMD1overexpression in GC tissues. GC patients with high intratumoral PSMD1 expression exhibit poorer survival than patients with low intratumoral PSMD1 expression. Our results demonstrate that PSMD1 expression is an independent predictor of GC patient outcomes. Integrated analysis of PSMD1 expression with TNM staging system provides a better prognostic model for GC patients and shows potential to aid both clinicians and patients in regard to counseling, individualized adjuvant treatment decision-making and follow-up scheduling.
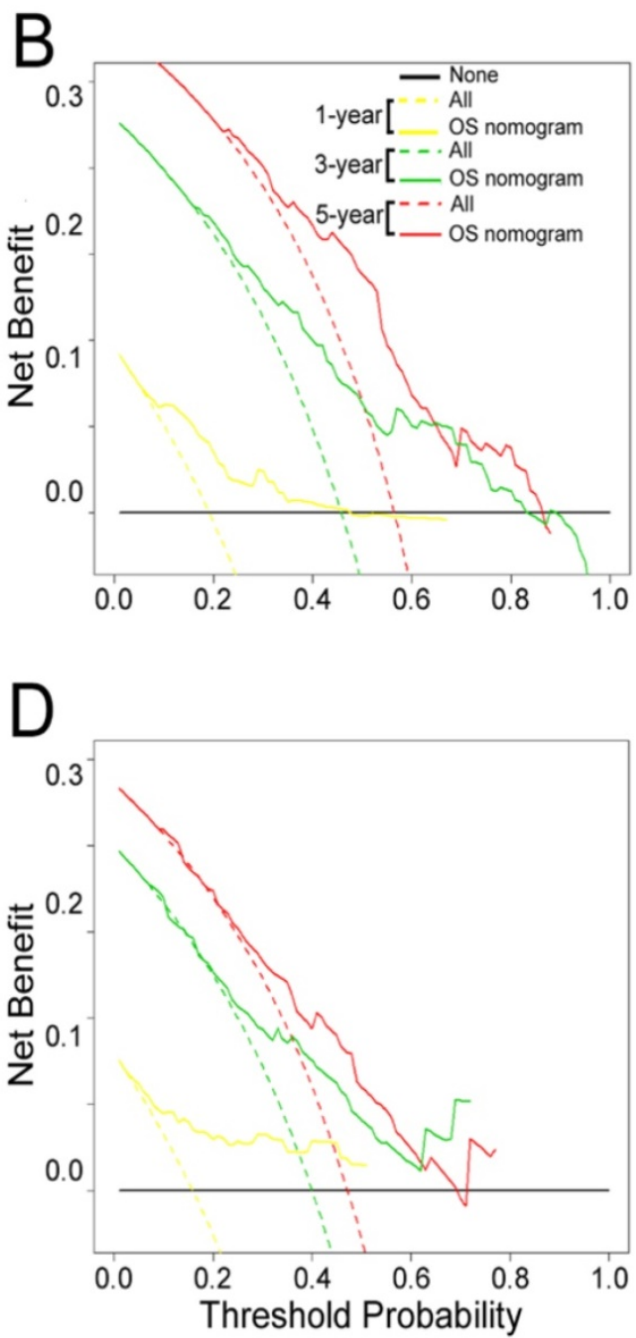

Figure 6. Decision curve analysis of the (A, C) DFS and (B, D) OS nomograms for the training and validation cohorts. The $y$-axis represents net benefit and the $x$-axis represents threshold probability. The solid lines (yellow, blue and red) represent the nomograms. The dotted lines (yellow, blue and red) represent the assumption that all patients survive for1-, 3-, or 5-years, respectively. The thin black line represents the assumption that none of the patients survive for 1-, 3-, or 5-years. The net benefit was calculated by subtracting the number of false positive patients from the number of true positive patients, taking into consideration the relative harm of foregoing treatment compared to the negative consequences of unnecessary treatment. ${ }^{15,16}$ Relative harm was calculated by [pt/( 1 -pt)]; "pt" represents the threshold probability where the expected benefit of treatment equals the expected benefit of avoiding treatment. The equation $([\mathrm{a}-\mathrm{c}] /[\mathrm{b}-\mathrm{d}]=[1 \mathrm{l} \mathrm{pt}] / \mathrm{pt})$ shows how a patient weighs the relative harms of false-positive and false-negative results at the time when he/she opts for treatment; ' $a-c$ ' represents the harm from a false-negative result; ' $b$ - $d$ ' represents the harm from a false-positive result; And a, b, c and d represent the values for true positive, false positive, false negative, and true negative results, respectively. ${ }^{15,16}$ Training cohort: (A, B). Validation cohort: (C, D). 


\section{Supplementary Material}

Supplementary figures and tables. http://www.jcancer.org/v10p4357s1.pdf

\section{Acknowledgments}

This work was supported by the grants from the State's Key Project of Research and Development Plan (2017YFC0108300, 2017YFC0108301), National Natural Science Foundation of China (81672446, 81270565), Natural Science Foundation of Guangdong Province (2016A030313843), Research Fund of Public Welfare in the Health Industry, the National Health and Family Planning Commission of China (201402015), the Southern Medical University Clinical Research Start-Up Project (LC2016ZD003), and the State's Key Clinical Specialty Discipline Construction Program([2011]170 ).

\section{Competing Interests}

The authors have declared that no competing interest exists.

\section{References}

1. Torre LA, Bray F, Siegel RL, Ferlay J, Lortet-Tieulent J and Jemal A. Global cancer statistics, 2012. CA: a cancer journal for clinicians. 2015; 65(2):87-108.

2. Razzak M. Genetics: new molecular classification of gastric adenocarcinoma proposed by The Cancer Genome Atlas. Nature reviews Clinical oncology. 2014; 11(9):499.

3. McLean MH and El-Omar EM. Genetics of gastric cancer. Nature reviews Gastroenterology \& hepatology. 2014; 11(11):664-674

4. Jiang Y, Xie J, Han Z, Liu W, Xi S, Huang L, et al. Immunomarker Support Vector Machine Classifier for Prediction of Gastric Cancer Survival and Adjuvant Chemotherapeutic Benefit. Clin Cancer Res. 2018; 24: 5574-5584.

5. Jiang $\mathrm{Y}, \mathrm{Li} \mathrm{T}$, Liang $\mathrm{X}, \mathrm{Hu} \mathrm{Y}, \mathrm{Huang} \mathrm{L}$, Liao Z, et al. Association of Adjuvant Chemotherapy With Survival in Patients With Stage II or III Gastric Cancer. JAMA Surg. 2017; 152: e171087.

6. Tomko RJ, Jr. and Hochstrasser M. Molecular architecture and assembly of the eukaryotic proteasome. Annual review of biochemistry. 2013; 82:415-445.

7. Mehta A, Zhang L, Boufraqech M, Zhang YQ, Patel D, Shen M and Kebebew E. Carfilzomib is an effective anticancer agent in anaplastic thyroid cancer. Endocr-Relat Cancer. 2015; 22(3):319-329.

8. Altmann A, Markert A, Askoxylakis V, Schoning T, Jesenofsky R, Eisenhut M and Haberkorn U. Antitumor effects of proteasome inhibition in anaplastic thyroid carcinoma. Journal of nuclear medicine : official publication, Society of Nuclear Medicine. 2012; 53(11):1764-1771.

9. Jonker PK, van Dam GM, Oosting SF, Kruijff $S$ and Fehrmann RS. Identification of novel therapeutic targets in anaplastic thyroid carcinoma using functional genomic mRNA-profiling: Paving the way for new avenues? Surgery. 2017; 161(1):202-211.

10. Okumura T, Ikeda K, Ujihira T, Okamoto K, Horie-Inoue K, Takeda S and Inoue S. Proteasome 26S subunit PSMD1 regulates breast cancer cell growth through p53 protein degradation. Journal of biochemistry. 2018, 163(1): 19-29.

11. Livak KJ and Schmittgen TD. Analysis of relative gene expression data using real-time quantitative PCR and the 2(-Delta Delta C(T)) Method. Methods. 2001; 25: 402-408.

12. Schmittgen TD and Livak KJ. Analyzing real-time PCR data by the comparative C(T) method. Nat Protoc. 2008; 3: 1101-1108.

13. Li TJ, Jiang YM, Hu YF, Li GX, et al. Interleukin-17-Producing Neutrophils Link Inflammatory Stimuli to Disease Progression by Promoting Angiogenesis in Gastric Cancer. Clinical cancer research : an official journal of the American Association for Cancer Research. 2017, 23(6): 1575-1585.

14. Jiang Y, Zhang Q, Hu Y, Li G, et al. ImmunoScore Signature: A Prognostic and Predictive Tool in Gastric Cancer. Annals of surgery. 2018, 267(3): 504-513.

15. Li T, Zhang $\mathrm{Q}$, Jiang $\mathrm{Y}, \mathrm{Yu}$ J, Hu Y, Mou T, Chen G and Li G. Gastric cancer cells inhibit natural killer cell proliferation and induce apoptosis via prostaglandin E2. Oncoimmunology. 2016; 5(2):e1069936.

16. Detre S, Saclani Jotti G and Dowsett M. A "quickscore" method for immunohistochemical semiquantitation: validation for oestrogen receptor in breast carcinomas. Journal of clinical pathology. 1995; 48(9):876-878.

17. Jiang Y, Liu W, Li T, Hu Y, Chen S, Xi S, et al. Prognostic and Predictive Value of p21-activated Kinase 6 Associated Support Vector Machine Classifier in
Gastric Cancer Treated by 5-fluorouracil/Oxaliplatin Chemotherapy. EBioMedicine. 2017; 22: 78-88

18. Collins GS, Reitsma JB, Altman DG and Moons KG. Transparent reporting of a multivariable prediction model for individual prognosis or diagnosis (TRIPOD): the TRIPOD statement. Annals of Internal Medicine. 2015; 162(10):735-6.

19. Liang WH, Zhang L, He JX, et al. Development and Validation of a Nomogram for Predicting Survival in Patients With Resected Non-Small-Cell Lung Cancer. Journal of Clinical Oncology. 2015; 33(8):861-869.

20. Vickers AJ, Cronin AM, Elkin EB and Gonen M. Extensions to decision curve analysis, a novel method for evaluating diagnostic tests, prediction models and molecular markers. BMC medical informatics and decision making. 2008; 8:53.

21. Localio AR and Goodman S. Beyond the usual prediction accuracy metrics: reporting results for clinical decision making. Annals of internal medicine. 2012; 157(4):294-295.

22. Camp RL, Dolled-Filhart M and Rimm DL. X-tile: a new bio-informatics tool for biomarker assessment and outcome-based cut-point optimization. Clinical cancer research : an official journal of the American Association for Cancer Research. 2004; 10(21):7252-7259.

23. Weiser MR, Gonen M, Chou JF, Kattan MW and Schrag D. Predicting survival after curative colectomy for cancer: individualizing colon cancer staging. Journal of clinical oncology : official journal of the American Society of Clinical Oncology. 2011; 29(36):4796-4802.

24. Ryu H, Gygi SP, Azuma Y, Arnaoutov A and Dasso M. SUMOylation of Psmd1 controls Adrm1 interaction with the proteasome. Cell reports. 2014; 7(6):1842-1848

25. Deng S, Zhou H, Yang H, et al. Over-expression of genes and proteins of ubiquitin specific peptidases (USPs) and proteasome subunits (PSs) in breast cancer tissue observed by the methods of RFDD-PCR and proteomics. Breast cancer research and treatment. 2007; 104(1):21-30.

26. Wang H, Liu S, Lu X, Zhao X, Chen S and Li X. Gene expression profile changes in NB4 cells induced by realgar. Chinese medical journal. 2003; 116(7):1074-1077.

27. Ching T, Song MA, Tiirikainen M, Molnar J, Berry M, Towner D and Garmire LX. Genome-wide hypermethylation coupled with promoter hypomethylation in the chorioamniotic membranes of early onset pre-eclampsia. Molecular human reproduction. 2014; 20(9):885-904.

28. Lagadec C, Vlashi E, Bhuta S, Lai C, Mischel P, Werner M, Henke M and Pajonk F. Tumor cells with low proteasome subunit expression predict overall survival in head and neck cancer patients. Bmc Cancer. 2014;: 14.152

29. Weiser MR, Landmann RG, Kattan MW, Gonen M, Shia J, Chou J, Paty PB, Guillem JG, Temple LK, Schrag D, Saltz LB and Wong WD. Individualized prediction of colon cancer recurrence using a nomogram. Journal of Clinical Oncology. 2008; 26(3):380-385. 